\title{
Enhanced double ionization with circularly polarized light
}

\author{
G. D. Gillen, M. A. Walker, and L. D. Van Woerkom \\ Department of Physics, The Ohio State University, 174 West 18th Avenue, Columbus, Ohio 43210-1106
}

(Received 31 May 2001; published 18 September 2001)

\begin{abstract}
Using 120-fs, 800-nm Ti:sapphire laser pulses, ionic yields of singly and doubly charged magnesium ions were measured as a function of intensity and laser field ellipticity. A clear "nonsequential" enhancement of the doubly charged ion is observed for circularly polarized light in addition to linearly polarized light. Over the entire intensity range the double-ionization yield is considerably higher for linear polarization than it is for circular polarization.
\end{abstract}

DOI: 10.1103/PhysRevA.64.043413 PACS number(s): 32.80.Rm, 32.80.Wr, 33.80.Rv, 33.80.Wz

\section{INTRODUCTION}

The identification of multielectron processes, including those responsible for multielectron detachment, has been the focus of many investigations for over two decades. The first reported measurements of multiply charged ion production via multiphoton ionization (MPI) were made by Aleksakhin et al. [1,2] in 1977 and 1979, with 1.06- $\mu \mathrm{m}, 30$-ns laser pulses. As laser technology advanced and shorter pulse durations became possible (also enabling higher peak intensities), new physical phenomena became observable, including high-order harmonic generation [3], above threshold ionization (ATI) [4], short-pulsed soft-X-ray sources [5], etc. A few years later, L'Huillier et al. reported ionic yield measurements for krypton and xenon using 1.064- $\mu \mathrm{m}$ 50-ps laser pulses $[6,7]$.

These later measurements revealed a much higher yield of multiply charged ions than expected according to a stepwise ionization process for the lower intensity range. A stepwise or sequential ionization process assumes that a singly charged ion is created by the ionization of a neutral atom; a doubly charged ion is created by the ionization of a singly charged ion, and so on. When displayed on a log-log scale of counts versus peak intensity, L'Huillier's measurements of the first ionic species initially grew linearly, following a power-law dependence for increasing ionization probability, as previously observed by Aleksakhin. As the intensity was increased the probability of ionization increased up to the saturation intensity. The saturation region starts when this probability approaches unity. In this intensity regime the number of neutral atoms in the ionization volume begins to be depleted and the singly charged ionic species is dominant. For full volume experiments (where the entire focal region along the axis of beam propagation is exposed to the detector) the ionization yield curve above the saturation limit is expected to grow as the Gaussian focal volume grows, by an $I^{3 / 2}$ rate. The enhancement of the double and triple ions observed by L'Huillier occurred for an intensity region below the saturation of the previous ionic species, contrary to what would be expected by sequential ionization models. A sequential model would not expect the second ionic species to be measurably detected until the ionic volume contains a significant number of singly charged ions. The observation of an unexplained "nonsequential" (NS) process sparked the interest of many investigations to come, both theoretical and experimental.

A decade after the first reported enhancement of doubly charged ionic species Fittinghoff et al. [8] observed a prominent enhancement of $\mathrm{He}^{2+}$ ions using 614-nm 120-fs laser pulses. The enhancement occurred in an intensity region where there should have been virtually no double ions as compared to their sequential Ammosov-Delone-Krainov (ADK) tunneling ionization model [9]. Soon after, Walker et al. [10], utilizing 780-nm 160-fs pulses, reported ionic yield measurements for helium over a vast detection and intensity range. The double ionization curve of this investigation illustrated an enhancement of the doubly charged ions several orders of magnitude above what would be expected for various sequential ionization models (ADK and SAE) below the saturation of the first ion. For peak laser intensities above this NS intensity region the number of ions detected started to increase again following the sequential models.

In this paper we present the results of strong-field ionization experiments with magnesium. Magnesium was chosen because it is a model two-electron atom with a moderately high first and second ionization potential, for an alkalineearth element. The first three ionization potentials for magnesium are 7.646, 15.035 , and $80.143 \mathrm{eV}$. A higher ionization potential is desired so that a larger number of photons are required to ionize the atom and results can be qualitatively compared to previous experiments of similar or higher MPI orders. There is also a lack of published experimental MPI or ATI studies of magnesium for Ti:sapphire laser frequencies. We report ionization yield curves of singly and double charged ions as a function of laser intensity and field ellipticity.

\section{EXPERIMENTAL SETUP}

The laser system used in this investigation is a Positive Light/Spectra Physics regeneratively amplified Ti:sapphire laser utilizing chirped pulse amplification (CPA) [11] with 120 -fs pulse duration and a $1-\mathrm{kHz}$ repetition rate with $0.9 \mathrm{~mJ}$ per pulse. The peak laser intensity in the interaction region was calibrated by collecting photoelectron spectra of argon for a variety of pulse energies. These spectra were then compared to spectra taken in a previous investigation [12] using the appearance of the eight-photon Freeman resonance of the $4 f$ Rydberg level as a calibration point. 


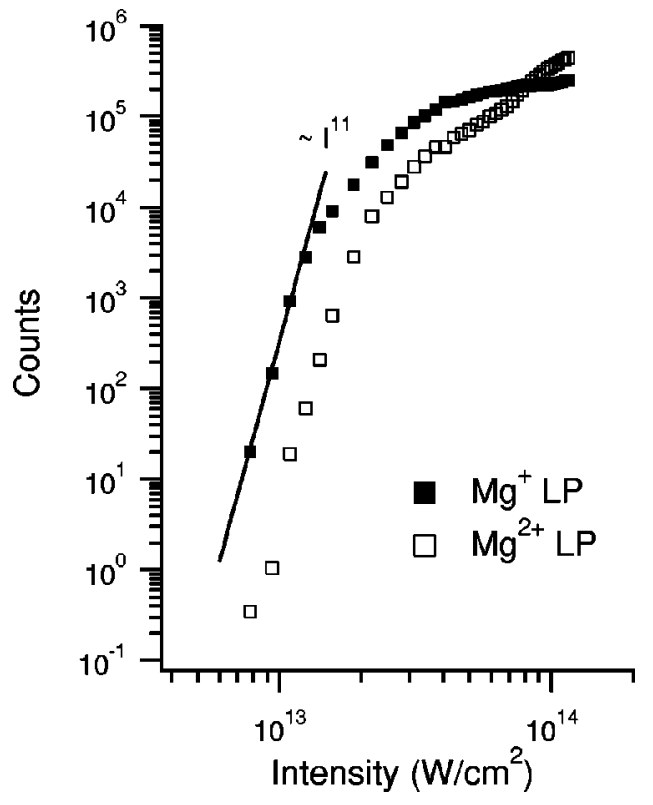

FIG. 1. Magnesium ionic yield curve for linear laser laser polarization.

The laser is focused into the vacuum chamber via a $25-\mathrm{cm}$ focal length lens with an initial beam diameter of $7.1 \mathrm{~mm}$ and propagates into the interaction region mutually orthogonal to the atomic beam and the ion flight path. The ellipticity of the laser field is controlled via a quarter-wave plate. Polarization is oriented such that when the field is linear it is parallel to the flight path. To reduce the effects of spatial averaging over the laser focus volume we can use a restricted volume technique [13] by placing a 1-mm pinhole in the flight path near the interaction region.

Each point is an integration of all of the counts detected for $\mathrm{Mg}^{+}$or $\mathrm{Mg}^{2+}$, including the isotopes, and normalized to one million laser shots per data point. Up to $10 \times 10^{6}$ shots were taken for lower yielding intensities for better statistics. The detection efficiencies of microchannel plates for various ions is proportional to $Z / M^{1 / 2}[14,15]$, where $Z$ is the charge of the ion and $M$ is its mass.

\section{RESULTS}

The measured ionic yield curves versus peak field intensity are displayed in Fig. 1 for linear polarization and in Fig. 2 for circular polarization. For both Figs. 1 and 2 some gross structural characteristics are apparent. On the log-log scale the detected yield of $\mathrm{Mg}^{+}$increases nearly linearly at low peak laser intensity followed by a decrease in slope until the saturation intensity is approached. For a restricted volume experiment, an iso-intensity volume for an intensity less than that of the peak does not continue to grow as quickly as it does for an unrestricted volume detection region, forcing the ion yield curve to flatten out for higher intensities. For a more detailed discussion of volume effects on the detected ionic yield see the appendix of Larochelle et al. [16].

The single ion yield for linear polarization (LP) has a power-law slope of about 11 for intensities up to about $1.5 \times 10^{13} \mathrm{~W} / \mathrm{cm}^{2}$ and then begins a gentle roll-off to satura-

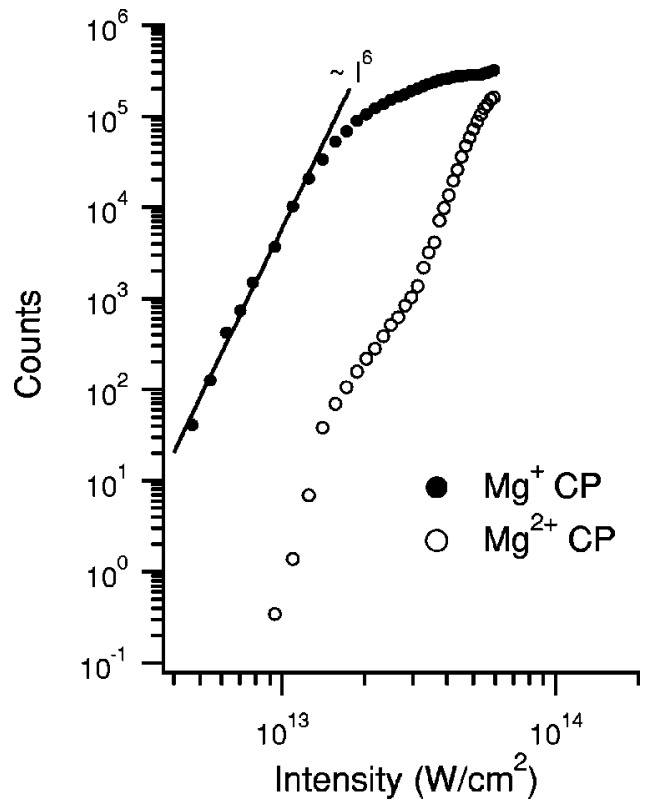

FIG. 2. Magnesium ionic yield curve for circular laser polarization.

tion at about $4 \times 10^{13} \mathrm{~W} / \mathrm{cm}^{2}$. It is clear that a simple powerlaw expression cannot describe the ion yield over the range $(1.5-4) \times 10^{13} \mathrm{~W} / \mathrm{cm}^{2}$. The $\mathrm{Mg}^{2+}$ yield increases initially up to a peak intensity around $(2-3) \times 10^{13} \mathrm{~W} / \mathrm{cm}^{2}$, then the rate decreases until an intensity of $6 \times 10^{13} \mathrm{~W} / \mathrm{cm}^{2}$. Above this intensity a secondary, and higher, ionization rate begins to take over until saturation of the interaction region is reached around $1 \times 10^{14} \mathrm{~W} / \mathrm{cm}^{2}$. One interesting result of using the restricted volume technique with a two-electron atom is that the number of doubly charged ions created can exceed that of the maximum number of singly charged ions. For a purely sequential ionization model, the disk-shaped ionization volume is dominated by singly charged ions at lower peak laser intensities. As the intensity is increased the total ionization volume grows in radius to the point where the central peak intensity is capable of double ionization. Further increases in the intensity will yield an annulus-shaped singly charged ionization volume, which does not grow in total volume for further increases in intensity, circumscribing the disk-shaped doubly charged ionic volume. Since the doubly charged ion is the highest charge state of the model its ionization volume will continue to grow beyond saturation as there is no higher charged state to deplete its central region. Any enhanced double ionization will blur the geometric borders between singly and doubly charged ionization volumes in favor of the double and hence increase the difference in their maxima as a function of intensity.

The use of circular polarization (CP) shows a clear powerlaw dependence for the single ion yield with a slope of 6 over the lower intensity range up to about $1.8 \times 10^{13} \mathrm{~W} / \mathrm{cm}^{2}$. Remarkably, the double ion yield persists below the saturation intensity for single ionization. An obvious "knee" structure is observed similar to that observed for linear polarization in a wide variety of noble-gas atoms $[10,16-18]$. Because the average pulse energy is the experi- 
TABLE I. Photon orders for three ionization processes.

\begin{tabular}{lc}
\hline \hline Multiphoton ionization order & Process \\
\hline $\operatorname{Mg}\left(3 s^{2}\right)+5 \gamma \rightarrow \mathrm{Mg}^{+}(3 s)+e^{-}$ & $(1)$ \\
$\operatorname{Mg}^{+}(3 s)+10 \gamma \rightarrow \mathrm{Mg}^{2+}+e^{-}$ & $(2)$ \\
$\operatorname{Mg}\left(3 s^{2}\right)+15 \gamma \rightarrow \mathrm{Mg}^{2+}+2 e^{-}$ & $(3)$ \\
\hline \hline
\end{tabular}

mentally controlled variable, the intensity axis for the points of Fig. 2 is shifted towards higher intensities by a factor of 2 to account for the peak field difference between the LP and $\mathrm{CP}$ for the same pulse energy.

The overall shapes of the $\mathrm{Mg}^{2+}$ ion yields for linear and circular polarization have similar characteristics. Overall, the largest difference in the double-ionization yield curves between linear and circular polarization is the pronounced suppression of double ionization for circular polarization. At the saturation intensity of $\mathrm{Mg}^{2+}$, linear and circular yields only differ by a factor of 3 . For lower intensities, in the region of the primary ionization rate, the yields differ by up to 3 orders of magnitude.

\section{DISCUSSION}

It is important to stress here that we are in a regime where the traditional Keldysh parameter $\gamma[19]$ would indicate that multiphoton ionization is the more dominant mechanism for the majority of peak laser intensities used. The minimum photon orders for each ionization process are shown in Table I. If $\gamma<1$, quasistatic tunneling ionization (as modeled by ADK) is accepted to be the dominant mechanism. If $\gamma>1$, MPI is the dominant mechanism. The Keldysh parameters for single and double ionization of magnesium are shown in Table II. For the majority of field strengths considered in this work all ionization should proceed above the Coulomb barrier and hence there exists no barrier for a tunneling mechanism. For an electric field of $1.3 \times 10^{13} \mathrm{~W} / \mathrm{cm}^{2}$ the field-free magnesium ground-state energy is equal to the saddle point of the Combined coulomb and laser potential, resulting in above the barrier ionization (ABI) [20].

Thus, magnesium presents an interesting case where ionization cannot be clearly labeled by a single simple process. Upper limits of the ionization rate for ABI can be calculated using ADK rates [20] and are found to fall in the range of 370 to $1 \mathrm{fs}^{-1}$ for intensities from 1.3 to $6 \times 10^{13} \mathrm{~W} / \mathrm{cm}^{2}$. Although the Coulomb barrier is greatly suppressed, the escape time for the electron is much longer than the optical period, and the quasistatic approximation fails. The dominant

TABLE II. Keldysh parameters for sequential processes of Table I for intensities in the lower and higher regions of this study.

\begin{tabular}{lcc}
\hline \hline Process & Intensity $\left(\mathrm{W} / \mathrm{cm}^{2}\right)$ & Keldysh parameter \\
\hline$(1)$ & $2 \times 10^{13}$ & 1.8 \\
$(1)$ & $1 \times 10^{14}$ & 0.8 \\
$(2)$ & $2 \times 10^{13}$ & 2.5 \\
$(3)$ & $1 \times 10^{14}$ & 1.1 \\
\hline \hline
\end{tabular}

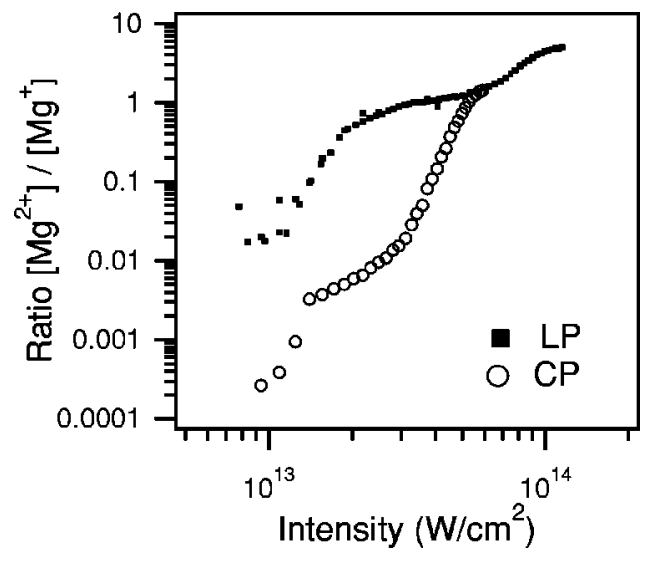

FIG. 3. Ratio of $\mathrm{Mg}^{2+} / \mathrm{Mg}^{+}$ionic yields versus intensity. The ratio fo LP contains additional points from those of Fig. 1 from a subsequent data run for the lower intensities.

ionization mechanism should be multiphoton in nature for the lower intensities and possibly contain character of ABI at higher intensities.

The enhancement of doubly charged ions for intensities lower than the saturation intensity of the singly charged ion is generally referred to as nonsequential double ionization (NSDI) [8]. Ionic yields are traditionally compared to calculated curves based upon ADK tunneling rates $[8,10,14,16,17]$ that assume the atom is entirely without structure, and the electron tunnels through the suppressed Coulomb barrier from the ground state of the neutral to the ground state of the ion. Sequential ADK ionization models do not predict any appreciable double ionization until the single ionization yield has reached its saturation intensity. Thus, any enhancement of the double ions above that predicted for a structureless atom has been referred to as "nonsequential" ionization.

A frequently used measure of nonsequential, or enhanced, double ionization is to plot the ratio of doubly charged ions to singly charged ions as a function of laser intensity $[10,14,16,18,21]$. Compared to the strong intensity dependence of observed ion yields the ratio of the double ion to the single ion in the enhanced, or nonsequential, region exhibits a low-order dependence on peak laser intensity. The dramatic difference of their intensity dependence shows that the number of singly and doubly charged ions is relatively independent of each other and the precursor to the double ion is not the single ion. Figure 3 displays the ionic ratios for magnesium for both linear and circular polarization of the laser field.

Although the exact ionization mechanism may not be clear, it is possible to address the enhanced double ionization of magnesium using four possible mechanisms: first and second generation rescattering models, the population of intermediate states via multiphoton absorption, and shake-off/ shake-up.

Rescattering has become an accepted mechanism responsible for observed enhanced double ionic yields for helium $[18,22-24]$ and a contributing mechanism in the other noble gasses [16]. The theory is based on the dynamics of an electron driven by the laser field after being born into the continuum [25]. As the electron's motion is governed mostly by 


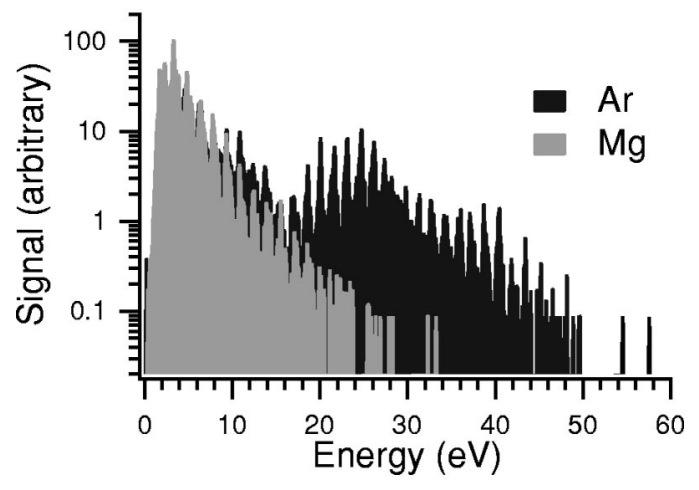

FIG. 4. Observed photoelectron spectra for argon and magnesium using the same experimental system and an intensity of $6.3 \times 10^{13} \mathrm{~W} / \mathrm{cm}^{2}$, with a pulse width of $120 \mathrm{fs}$.

the oscillating laser electric field it can reencounter its parent ion where an elastic or inelastic collision can take place. Elastic collisions are responsible for the production of high energy or "plateau" electrons, while inelastic collisions produce ionic excitations or possibly ionization, resulting in the observed enhanced double ionization. Theoretical calculations using the rescattering model predict a plateau, or extension of photoelectron spectra (PES) beyond the $2.5 U_{p}$ cut off predicted for tunneling ionization in the absence of rescattering [26], where $U_{p}$ is the ponderomotive energy [27]. With rescattering, this plateau extends out to a cut-off energy of $10 U_{p}$ [26]. The model used by Lohr et al. [26] also predicts a MPI cut-off energy of $4.5 U_{p}$. Extensions of PES energies are frequently observed for $\mathrm{He}[10,18,21]$ and the noble gasses $[12,21,28]$.

No extension of photoelectron energies is observed for magnesium, as illustrated in Fig. 4, showing spectra of both magnesium and argon, measured using the same experimental system, laser intensity, and pulse width. The ponderomotive energy for an intensity of $6.3 \times 10^{13} \mathrm{~W} / \mathrm{cm}^{2}$ is $4 \mathrm{eV}$, giving a MPI cut off at $18 \mathrm{eV}$ and a rescattering cut off at $40 \mathrm{eV}$, which agrees well with $\mathrm{Mg}$ and Ar PES, respectively. The plateau for argon is both calculated and observed [12] to be anomalously high compared to helium $[10,21]$ and neon [21], where they occur between 3 and 6 orders of magnitude below the signal of the first few ATI orders. It is possible that magnesium PES may have a plateau occurring at a level not detectable at this point. Furthermore, if the majority of rescattering events produced inelastic collisions, no high-energy signature would persist. Thus, while the data are suggestive of no rescattering, it cannot be ruled out completely.

In addition to hot-electron production, the rescattering model predicts a strong ellipticity dependence for the double ionization. Generation of high-order harmonics, for example, is a phenomenon based on rescattering that relies upon the freed electron reencountering its parent ion. In 1993, Budil et al. observed that emission of harmonics is highly dependent upon the driving field ellipticity [29]. As laser ellipticity approached only 0.2 , emission of harmonics dropped by two to three orders of magnitude. Only the slightest degree of ellipticity is required for the electron wave packet to be steered away from a direct collision with the ion [22]. Lohr et al. reported measurements of photoelectrons near the

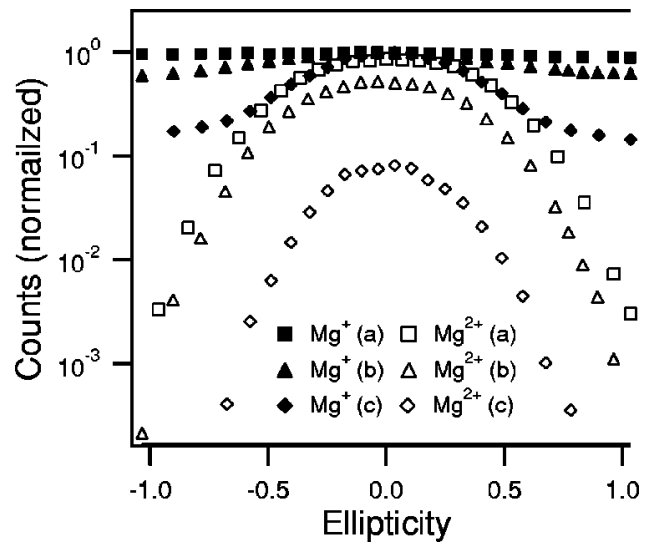

FIG. 5. Ellipticity dependence of singly and doubly charged magnesium ions for LP intensities of (a) $5.0 \times 10^{13}$, (b) $2.9 \times 10^{13}$, and (c) $1.5 \times 10^{13} \mathrm{~W} / \mathrm{cm}^{2}$. The total field energy is held constant as the ellipticity is changed.

$10 U_{p}$ cut off for neon drop by 2 orders of magnitude for ellipticities between 0.3 and 0.4 [26]. Figure 5 shows a plot of ionic yield measurements for magnesium as a function of the ellipticity of the laser field for LP intensities of 1.5, 2.9, and $5.0 \times 10^{13} \mathrm{~W} / \mathrm{cm}^{2}$. For these plots the total field energy is kept constant while the ellipticity of the field is adjusted. A strong dependence on the field ellipticity is observed for the double ion, but not comparable to that observed for helium and neon. In order for the double ion yield to drop by two orders of magnitude for a laser intensity on the lower end of the enhanced region an ellipticity of roughly 0.7 , or greater, is required.

The fact that we measure a clear double-ionization enhancement for the $\mathrm{CP}$ raises interesting questions. The only other measurement showing this effect for the $\mathrm{CP}$ is the recent work of Guo using the diatomic molecule NO [30]. Semiclassical trajectory calculations for the noble gases and the $\mathrm{CP}$ show that the electron excursion is quite large [31]. We estimate a return impact parameter on the order of 6-7 atomic units with a wave packet spreading of about 6 a.u. A reasonable estimate of the size of the magnesium ion ground state is approximately 1-2 a.u. which could produce enough "soft" collisions with the returning electron to excite the ion to the $3 p$ state. Ionization of the $3 p$ ion proceeds with nearly the same rate as the neutral and thus gives an enhancement of the double ionization below the saturation of the single ionization process for the $\mathrm{CP}$.

One shortfall of the first generation rescattering model to explain enhanced, or nonsequential, ionization is the assumption that the atom is completely without structure. The cutoff intensity for the laser field to provide enough energy to the freed electron in order to ionize the ion from the ground state is typically higher than the observed region of nonsequential ionization. Walker et al. reported this in the benchmark nonsequential experiment [10]. The cut-off intensity for magnesium and 800-nm photons would be $7 \times 10^{13} \mathrm{~W} / \mathrm{cm}^{2}$, which is also higher than the observed enhanced double ionization region.

Second generation rescattering theory models have not only incorporated collisional excitations of the ion into 


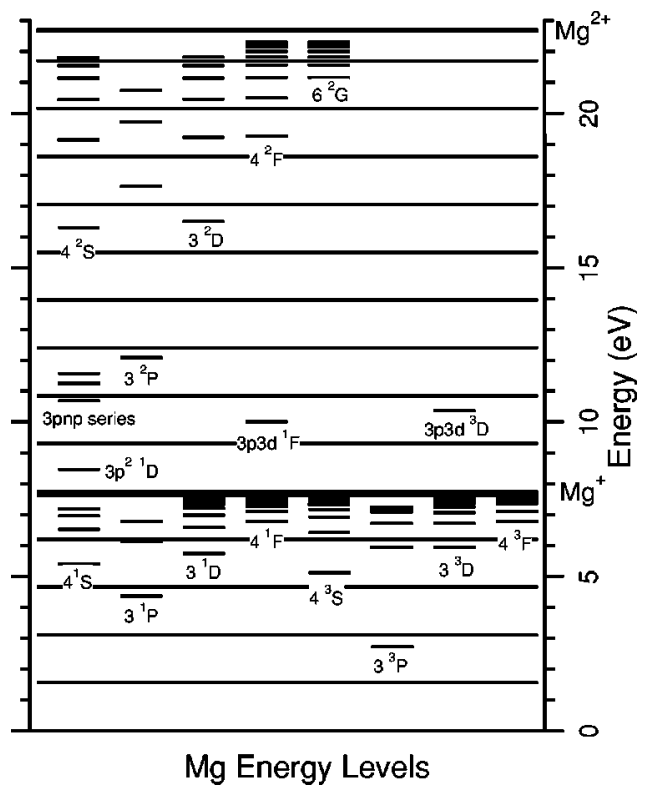

FIG. 6. Energy-level diagram for magnesium. The full length horizontal lines correspond to integer photon multiple energies for 800 -nm light. Ionization potentials of the neutral and first ion are $7.646 \mathrm{eV}$ and $15.035 \mathrm{eV}$, respectively.

higher states allowing subsequent ionization via a lowerorder process [23], but also include Coulomb focusing, or trapping, of the oscillating free electron by the Coulomb potential well of the ion [18]. The former model improved the agreement between experiment and calculation for $\mathrm{Kr}, \mathrm{Xe}$ [17], and Ar [16], but still falls up to a couple orders of magnitude lower than measured ratios of the single to double ion. The latter model allowed for excellent agreement for $\mathrm{He}$ [18]. Although, at this time the ellipticity dependence for these models is unknown, their collision-based principles exclude them as dominant-enhanced ionization mechanisms for circular and near-circular fields.

Population of intermediate states via multiphoton absorption must be considered as a probable dominant mechanism for magnesium with 800 -nm light. Magnesium has a very rich electronic structure as illustrated in Fig. 6. For linearly polarized light the number of parity-allowed transitions, and subsequent ionization paths through intermediate states, is considerably large. Table III indicates some of the stronger

TABLE III. Some of the possible transitions for LP light near an integer photon resonance and their relative photon orders.

\begin{tabular}{lc}
\hline \hline Transition & Photon order \\
\hline $\mathrm{Mg}\left(3 s^{2}\right) \rightarrow 3 s 3 p$ & 3 \\
$\mathrm{Mg} 3 s 3 p \rightarrow 3 s 3 d$ & 1 \\
$\mathrm{Mg}^{+} 3 s \rightarrow 3 p$ & 3 \\
$\mathrm{Mg}^{+} 3 p \rightarrow 4 s$ or $3 d$ & 3 \\
$\mathrm{Mg}^{+} 4 s \rightarrow 4 p$ & 1 \\
$\mathrm{Mg}^{+} 4 p \rightarrow 5 s$ or $4 d$ & 1 \\
\hline \hline
\end{tabular}

possible transitions that are near-integer photon energies apart. If any of the lower ionic excited states are significantly populated then the new effective ionization limit, and photon orders necessary to ionize them, becomes comparable to that of the neutral states. Ionization from the $3 p$ ionic state requires $10.6 \mathrm{eV}$, from the $4 s$ state $6.4 \mathrm{eV}$, and from the $3 d$ state $6.2 \mathrm{eV}$. Ionization rates of excited ions would then be similar to that of neutral magnesium atoms. Subsequently, the observed ionization yields for singly and doubly charged ions would have similar slopes forcing a plot of their ratio to flatten out.

The importance of including magnesium's rich atomic structure when considering enhanced double ionization was made evident by the investigation of Trainham et al. [32] for a "direct" channel for NSDI of magnesium. Their investigation led them to believe that isolated core excitation (ICE) and nonresonant multiphoton transitions would lead to lowlying excited states of the ion and doubly excited autoionizing states and not a direct channel. As mentioned above, excited ions would have similar ionization rates and intensity dependences to that of neutrals. Xenakis et al. [33] observed a less pronounced enhancement of double ionization of magnesium for LP light using 400-nm photons and attributed the enhancement to atomic structure, or resonances and channel closings.

Magnesium atoms exposed to 800 -nm circularly polarized light essentially look like structureless atoms. For ionization of the neutral, there are virtually no accessible excited states due to angular momentum selection rules. The only available states are $6 h$ and higher angular momentum states. Such states would be Rydberg states ponderomotively shifted into resonance with five or six photons. For ionization of the ion, the states would have to have an angular momentum of 10 or higher. All of these states of the neutral and the ion reside very close to their respective ionization limits. Thus, all of the probable ionization paths through intermediate states available for the LP are essentially closed for the CP. As the polarization changes from linear to circular, the number of accessible intermediate states falls off slowly at first. Thus, an ellipticity dependence for multiphoton transitions is expected to be less severe than for the rescattering case.

Finally, it is possible for the rapid departure of the first electron to create a change in the potential for the remaining electron that is sufficient to shake it into excited ionic states. As pointed out earlier, any ionic excitation reduces the effective ionization order for the ion to that of the neutral. Shake up is a well-known phenomenon in the alkaline-earth atoms [34] and in single photon x-ray excitation of atoms [35]. Shakeup exhibits no strong ellipticity dependence and hence does not preclude the production of enhanced double ionization for circular polarization.

\section{CONCLUSION}

We have measured single and double ionization yields for magnesium using 800-nm intense laser light and both linear and circular polarization. Enhanced double ionization is ob- 
served for both linear and circular polarization. It is not clear that conventional rescattering theory can explain the ellipticity dependence of the data and quantitative calculations must be performed. The persistence of enhanced double ionization for circular polarization contradicts simple rescat -tering theories and opens the possibility for studying other ionization mechanisms.

The authors would like to thank P. Corkum for helpful discussions. This work was supported by the NSF under Grant No. 9876965.
[1] I.S. Aleksakhin, I.P. Zapesochnyi, and V.V. Suran, JETP Lett. 26, 11 (1977).

[2] I.S. Aleksakhin, N.B. Delone, I.P. Zapesochnyi, and V.V. Suran, Sov. Phys. JETP 49, 447 (1979).

[3] R.M. Potvliege and R. Shakeshaft, Phys. Rev. A 40, 3061 (1989).

[4] P. Agostini, F. Fabre, G. Mainfray, G. Petite, and N.K. Rahman, Phys. Rev. Lett. 42, 1127 (1979).

[5] M.H. Sher, U. Mohideen, H.W.K. Tom, O.R. Wood II, G.D. Aumiller, R.R. Freeman, and T.J. McIlrath, Opt. Lett. 18, 646 (1993).

[6] A. L'Huillier, L.A. Lompre, G. Mainfray, and C. Manus, Phys. Rev. Lett. 48, 1814 (1982).

[7] A. L'Huillier, L.A. Lompre, G. Mainfray, and C. Manus, Phys. Rev. A 27, 2503 (1983).

[8] D.N. Fittinghoff, P.R. Bolton, B. Chang, and K.C. Kulander, Phys. Rev. Lett. 69, 2642 (1992).

[9] M.V. Ammosov, N.B. Delone, and V.P. Krainov, Sov. Phys. JETP 64, 1191 (1986).

[10] B. Walker, B. Sheehy, L.F. DiMauro, P. Agostini, K.J. Schafer, and K.C. Kulander, Phys. Rev. Lett. 73, 1227 (1994).

[11] D. Strickland and G. Mourou, Opt. Commun. 56, 219 (1985).

[12] M.J. Nandor, M.A. Walker, L.D. Van Woerkom, and H. G. Muller, Phys. Rev. A 60, R1771 (1999).

[13] M.A. Walker, P. Hansch, and L.D. Van Woerkom, Phys. Rev. A 57, R701 (1998).

[14] C. Guo, M. Li, J.P. Nibarger, and G.N. Gibson, Phys. Rev. A 58, R4271 (1998).

[15] C. Cornaggia, J. Lavancier, D. Normand, J. Morellec, and P. Agostini, Phys. Rev. A 44, 4499 (1991).

[16] S. Larochele, A. Talebpour, and S.L. Chin, J. Phys. B 31, 1201 (1998).

[17] A. Talebpour, C.-Y. Chien, Y. Liang, S. Larochelle, and S.L. Chin, J. Phys. B 30, 1721 (1997).

[18] V.R. Bhardwaj, S.A. Aseyev, M. Mehendale, G.L. Yudin, D.M. Villeneuve, D.M. Rayner, M.Yu. Ivanov, and P.B. Corkum,
Phys. Rev. Lett. 86, 3522 (2001).

[19] L.V. Keldysh, Sov. Phys. JETP 20, 1307 (1965). The Keldysh parameter is $\left(\mathrm{IP} / 2 U_{p}\right)^{(1 / 2)}$, where IP is the ionization potential and $U_{p}$ is the ponderomotive energy.

[20] A. Scrinzi, M. Geissler, and T. Brabec, Phys. Rev. Lett. 83, 706 (1999).

[21] B. Sheehy, R. Lafon, M. Widmer, B. Walker, L.F. DiMauro, P.A. Agostini, and K.C. Kulander, Phys. Rev. A 58, 3942 (1998).

[22] P.B. Corkum, Phys. Rev. Lett. 71, 1994 (1993).

[23] M.Yu. Kuchiev, J. Phys. B 28, 5093 (1995).

[24] M. Lein, E.K.U. Gross, and V. Engel, Phys. Rev. Lett. 85, 4707 (2000).

[25] M.Yu. Kuchiev, JETP Lett. 45, 404 (1987).

[26] A. Lohr, W. Becker, and M. Kleber, in Multiphoton Processes 1996, edited by P. Lambropoulos and H. Walther, Int. Conf. Ser. No. 154 (IOP, Bristol, 1997), p. 92.

[27] The ponderomotive energy $U_{p}$ is defined in atomic as $I /\left(4 w^{2}\right)$, where $I$ is the laser intensity and $w$ is the laser frequency.

[28] P. Hansch, M.A. Walker, and L.D. Van Woerkom, Phys. Rev. A 55, R2535 (1997).

[29] K.S. Budil, P. Salières, A. L'Huillier, T. Ditmire, and M.D. Perry, Phys. Rev. A 48, R3437 (1993).

[30] C. Guo and G.N. Gibson, Phys. Rev. A 63, 040701 (2001).

[31] M. Protopapas, D.G. Lappas, and P.L. Knight, Phys. Rev. Lett. 79, 4550 (1997).

[32] R. Trainham, N.J. Van Druten, L.D. Noordam, H.G. Muller, P. Breger, G. Petite, E. Mevel, P. Agostini, and A. Antonetti, in Coherence Phenomena in Atoms and Molecules in Laser Fields, edited by A.D. Bandrauk and S.C. Wallace (Plenum Press, New York, 1992).

[33] D. Xenakis, N.E. Karapanagioti, D. Charlambidis, H. Bachau, and E. Cormier, Phys. Rev. A 60, 3916 (1999).

[34] T.F. Gallagher, Rydberg Atoms (Cambridge University Press, Cambridge, 1994), p. 434.

[35] T. Åberg, Phys. Rev. 156, 35 (1967). 